\title{
豚鼠胰腺内钙调素的免疫组 织化学定位研究
}

徐天东 苏慧慈 黄威权

(第四军医大学组织学与还胎学教研空, 西安 710032)

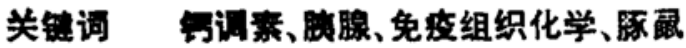

钻离子是重要的细胞内调节因子. 它的作用几乎涉及到所有的细胞生理过程，如物质代 谢、激素分泌、神经递愿的合成与释放、肌肉收缩以及细胞的分裂增殖等. 钙调素(calmodulin, $\mathrm{CaM}$ ）是一种广泛分布于真核细胞中的小分子蛋白,它作为细胞内主要的钻受体，传递钙离子 浓度变化的信息，影响许多关键酶的活性和生理过程的速率 ${ }^{[1]}$. 有研究表明, CaM 在神经组 织、睪丸和各种内分泌组织中含量丰富 ${ }^{[2]}$. 我们曾用免疫组织化学法观察到 $\mathrm{CaM}$ 广泛分布于 豚鼠留和小肠粘膜的内分泌细胞中 ${ }^{[2]}$. 已发现 $\mathrm{CaM}$ 与胰岛 $\beta$ 细胞的功能有密切关系 ${ }^{[3]}$. 然而 对费腺内 $\mathrm{CaM}$ 的分布与定位迄今仍缺乏详细研究. Seto-Ohshima 等采用甲醛固定组织，以 及作者用 Bouin 液固定组织, 进行胰腺 CaM 免疫组织化学研究, 均未能显示腌岛细胞是否存 在 $\mathrm{CaM}^{[2,4]}$. 本文用 $4 \%$ 多聚甲醛和 $2.5 \%$ 戊二酫联合固定并结合硼氢化钠 $\left(\mathrm{NaBH}_{4}\right)$ 处理的 方法, 研究了胰腺中 $\mathrm{CaM}$ 的定位, 证明了䀠鼠胰岛和胰腺间质副交感神经节中均有 $\mathrm{CaM}$ 免 疫反应阳性细胞.

\section{1 材料与方法}

\section{1 试剂}

兔抗 $\mathrm{CaM}$ 抗体系日本爱知发生研究院水谷昭教授惠赠 ${ }^{[5,} \mathrm{ABC}$ 药盒为 Vector 公司产品.

\section{2 材料}

正常成年豚鼠 8 只,雌雄不拘, 体重 300-450g, 断头处死. 取胰腺固定于含 $4 \%$ 多聚甲 醛、2.5\% 戊二醛和 $0.05 \% \mathrm{CaCl}_{2}$ 的固定液中, 过夜, 常规石蜡包理. 制成 $3 \mu \mathrm{m}$ 厚切片, 裱贴 于预先涂有铬矾明胶的载玻片上,烤干备用.

\section{3 免疫组织化学染色程序}

兔抗 $\mathrm{CaM}$ 抗体稀释度为 $1: 100$, 生物素标记的猖抗兔 $\operatorname{IgG}$ 的稀释度为 $1: 500, \mathrm{ABC}$ 复合 物的稀释度为 $1: 100$. 切片按常规法脱蜡至水, 当下行至 $50 \%$ 酒精时, 经 $\mathrm{NaBH}_{4}(0.5 \mathrm{mg} / \mathrm{ml}$ $50 \%$ 酒精)处理 $10 \mathrm{~min}$, 其余步骤按 $A B C-G D N$ 技术进行 ${ }^{[2]}$. 对照试验包括:（1）替代对炤 (用正常兔血清代替第一抗体);(2) 空白对照(以 PBS 代替第一抗体)。

\section{2 实验结 果}

$\mathrm{CaM}$ 免疫反应（CaM-IR）产物呈蓝黑色,背底着色浅, 阳性反应很易观察。本实验的阙 种对照试验均呈阴性反应。

1993-02-16 收稿,1993-05-28 收綮改稿. 
含 $\mathrm{CaM}$ 的细胞主要为胰岛细胞, 胰腺腺泡细胞呈阴性反应(图 1-1), 肤腺间质副交感神 经节内有 $\mathrm{CaM}-\mathrm{IR}$ 神经细胞分布(图 1-2). 在费岛中, CaM-IR 细胞分布于整个胰岛(图 1-1, $3,4)$. 细胞形状多样, 以圆形和多边形居多. 大部分细胞体积较小. 有些细胞体积则较大, 并 且有突起伸向相邻细胞之间, 呈现旁分泌的形态特征(图 1-3,4). CaM-IR 产物存在于胰岛细: 胞胞浆内,胞核为阴性. 此外, CaM-IR 强度在各肤岛及不同的胰岛细胞间存在差异. 多数细. 胞着色深且均匀,少数细胞浅淡(图 1-1,3,4). 在间质副交感神经节内, CaM-IR 神经细胞呈. 椭圆形或多角形, 聚集成群, 细胞界限不清楚. 胞质呈强阳性反应, 核为阴性反应(图 1-2).

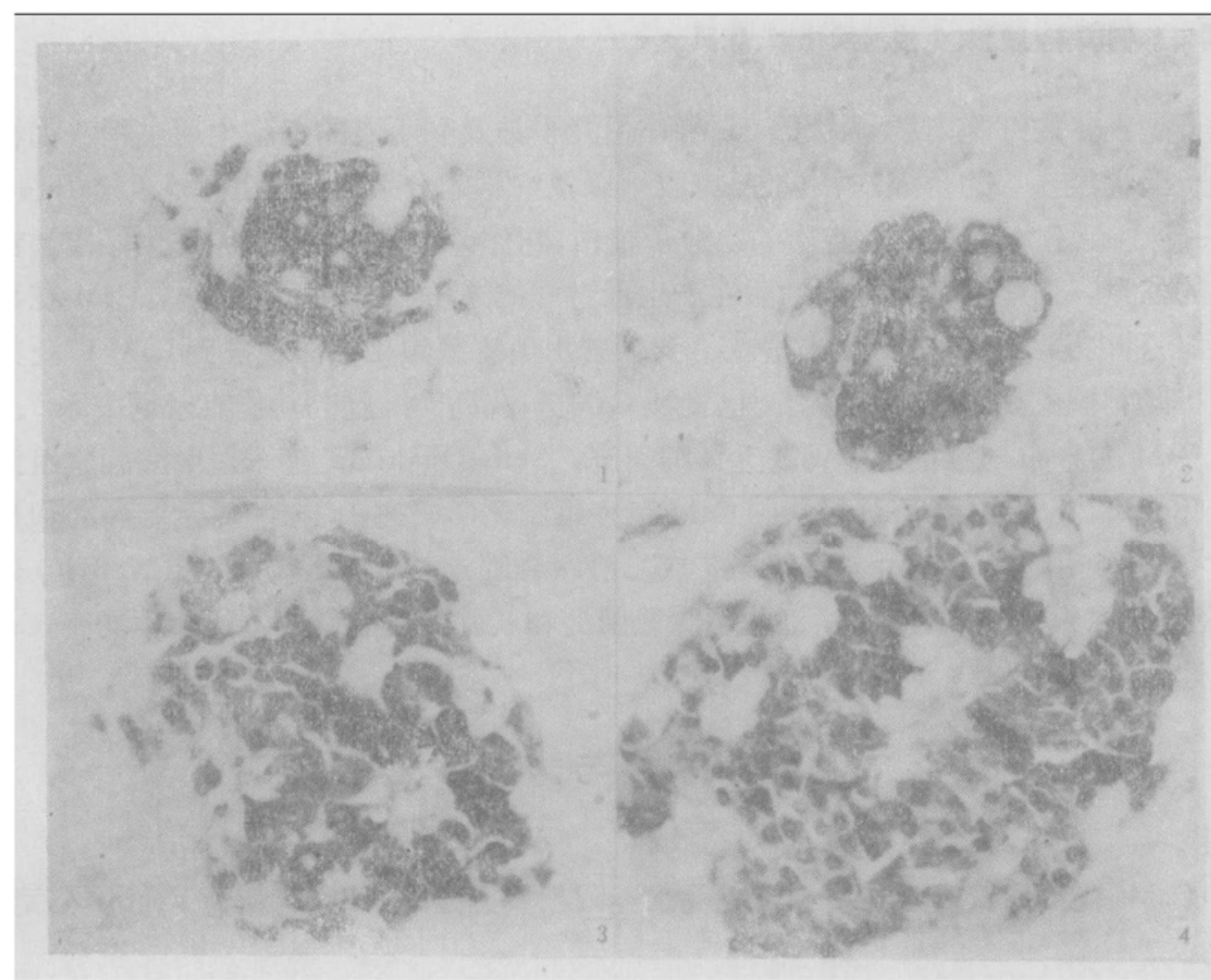

图 1

1.渎舟内 $\mathrm{CaM}_{a}$ 免度反应细胞的分布. 膄腺腺泡呈阴性反应. $\times 200$;

2.陵腺间质,示 $\mathrm{CaM}$ 免良反应神经细胸,胸质充满 CaM-IR 产物,胸核呈阴性. $\times 350$;

3. $\mathrm{C}_{\mathrm{a}} \mathrm{M}-\mathrm{IR}$ 细胞分布于整个费为内, 各细胞的 $\mathrm{CaM}-\mathrm{IR}$ 强度不等. 介示细胸有突起伸 向相邻细胞之间. $\times 350$;

4.腾舟内的 CaM-IR 细跑,形状多样,大小不等. $\times 400$

\section{3 讨 论}

我们应用 $4 \%$ 多聚甲醛和 $2.5 \%$ 戊二酫混合液固定并结合 $\mathrm{NaBH}_{4}$ 处理的方法, 对䀠鼠 胰腺内 CaM-IR 细胞的分布及形态作了初步观察. 结果显示, 胰息和胰腺间质副交感神经节 均存在 CaM-IR 细胞.

近年来,免疫组织化学技术已成为研究细胞内 $\mathrm{CaM}$ 定位的常用手段. 然而, 在免疫组织 化学染色过程中, 有许多因素影响其结果, 如固定不当造成抗原从原位弥散或抗原变性, 干扰 了准确定位和结哭分析. 在本研究中, 我们采用 $4 \%$ 多聚甲醛加上高浓度戊二醛的混合固定 液固定组织, 主要理由是: 先由固定作用较弱的甲醛快速渗人组织将之初步固定, 随后再由治 
定作用较强的戊二酫逐渐渗人加强固定, 以确保组织结构和保存抗原. 然而戊二醛的两个游 离醀基对抗原性损伤大. 这是因为戊二醛与蛋白质之间是以双键连接的，这种连接方式可能 在一定程度上破坏蛋白质的空间结构, 导致抗原性改变 ${ }^{66}$. 此外, 用高浓度戊二醀固定还可能 由于许多与非抗原成分结合的戊二酴分子仅有一个醴基与氨基发生反应,另一个醛基游离, 因 而抗体可以与这些游离醛基结合可造成背底的非特异性染色. 近年来的一些研究表明的，高 浓度戊二醛固定后的组织再经 $\mathrm{NaBH}_{4}$ 处理即可恢复抗原性. 因为 $\mathrm{NaBH}_{4}$ 能有效地将戊二 酫和蛋白质间的双键还原为单键. 双键的解离则使: (1) 组织和抗原的空间结构得以恢复从 而恢复抗原性 ${ }^{[6,7]}$; (2)消除了游离醛基而使非特导性背底染色大为减低. 我们实验的结果表 明, 本方法明显优于其它固定方法, 可有效地去除背底染色, 并显示了一些用常规甲醛固定液 未能显示 ${ }^{[2,4]}$ 的阳性结果.

关于 $\mathrm{CaM}$ 与胰忽的关系, 近来已有许多研究 ${ }^{[3,8,9]}$. 1979 年 Sugden 等首先报道, 胰离细 胞含有丰富的 $\mathrm{CaM}^{[8]}$. 两年后, Hutton 等从大鼠胰岛肿㿔细胞中分离出 $\mathrm{CaM}$, 并鉴定了其氨 基酸组成及与 $\mathrm{Ca}^{2+}$ 的结合能力 ${ }^{[9]}$. 本文的观察显示, CaM-IR 细胞分布在整个胰岛. 从细胞分 布和形态特点看, 这些细胞大多数可能是 $\mathrm{B}$ 细胞, 可能还有 $\mathrm{A}$ 细胞和 $\mathrm{D}$ 细胞. 因此, $\mathrm{CaM}$ 可能 与䐂岛 $A, B, D$ 等细胞的功能有密切关系. 有学者用分离的胰岛进行体外细胞培养, 发现 $\mathrm{CaM}$ 拮抗剂三氟吡啦嗪 (trifluoperazine, TFP) 明显抑制胰岛素的释放 ${ }^{[10,11]}$, 说明 CaM 在胰 岛素释放过程中可能起重要作用. 事实上,胰岛内的 $\mathrm{CaM}$ 也如同其它组织一样, 对腌岛具有 多种调节功能, 如激活环核苩酸磷酸二酯酶、腺苷酸环化酶、 $\mathrm{Ca}^{2+}-\mathrm{ATP}$ 酶和蛋白激酶等多种 酶的活性, 参与 $\beta$ 细胞内 $\mathrm{c}^{\prime} . \mathrm{MP}$ 和 $\mathrm{Ca}^{2+}$ 浓度的调节 ${ }^{[3]}$. $\mathrm{CaM}$ 还影响胰岛素前体的生物合

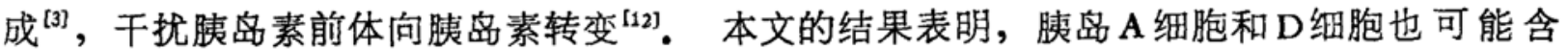
$\mathrm{CaM}$, 但有待进一步证实. 至于 $\mathrm{CaM}-\mathrm{IR}$ 强度在不同胰岛及各胰岛细胞的差异, 可能反映了 细胞功能状态的差异.

许多研究证实, 大脑皮质、小脑皮质、丘脑、下丘脑、海马、脑神经核和脊髓前角运动神经元 等均含有丰富的 $\mathrm{CaM}^{[13]}$. $\mathrm{CaM}$ 不仅调节突触传递、参与神经递质的合成与释放, 还参与轴浆 运输和神经元许多蛋白质磷酸化。我们观察到胰腺间质副交感神经节内有 CaM-IR 神经元, 提示 $\mathrm{C}_{3} \mathrm{M}$ 与这些神经元的功能有关.

\section{参紊献}

[1] Means, A. R., Tash, J. S., Chafouleas, J. G., Physiol. Rev., 1982, 62(1):1-39.

[2] 徐天乐、苏慧慈, 解剖学报, 1993, 24(2): 187-190.

[3] Valverde, I., Malaisse, W. J., Experientia, 1984, 40(10): 1061-1068.

[4] Seto-Ohshima, A., Sano, M., Mizutani, A., Acta Histochem. Cytochem., 1985, 18(3):275 - 282.

[5] Kitajima, S., Seto-Ohshima, A., Sano, M. et al., J. Biochem. (Takyo), 1983, 94(2):559 -. 564.

[6] Eldred, W. D., Zucker, C., Karten, H. J. et al., J. Histochem. Cyzochem., 1983, 31(2):285-292.

[7] Willingham, M. C., J. Histochem. Cytochem., 1983, 31(6):791-798.

[8] Sugden, M. C., Christie, M. R., Ashcroft, S. J. H., FEBS Letz., 1979. 105:95-100.

[9] Hutton, J. C., Penn, E. J., Jackson, P. e: al., Biochem. J., 1981, 193: 875-885.

[10] Krausz, Y., Wollheim, C. B., Siegel, E. et al., J. Clin. Invest., 1980, 66:603-607.

[11] Valverde, I., Sener, A., Herchuelz, A. et al., Endocrinology, 1981, 108:1305-13:2.

[12] Niki, H., Niki, A., Hidaka, H., Biomed. Res., 1981, 2:413-417.

[13] 徐天乐、黄威衩、苏㥿慈等，科学通报，1993，38(7): 650--652. 